\title{
Molecular Studies of Candidate Gene Somatic Mutations in Human Schwannoma Tumors
}

\author{
Amanda V. Martinez ${ }^{1}$, Hua $\mathrm{Li}^{2}$, and Margaret Wallace ${ }^{2}$ \\ ${ }^{1}$ College of Medicine, University of Florida \\ ${ }^{2}$ Department of Molecular Genetics and Microbiology, University of Florida
}

Faculty mentor: Margaret Wallace, Department of Molecular Genetics and Microbiology

\begin{abstract}
Schwannomas are benign Schwann cell tumors on the peripheral nerves that occur in the autosomal dominant conditions of NF2 (neurofibromatosis type 2) or schwannomatosis. NF2 is caused by germline mutations in the NF2 gene, and patients can form tumors when the remaining normal gene copy is mutated. In schwannomatosis, two genes are known to have germline mutations: SMARCB1 and LZTR1, both near the NF2 gene on chromosome 22. Schwannomas in schwannomatosis have variable somatic deletions of multiple genes, and the tumors also have a risk of becoming malignant. These tumors can cause deformities, functional problems, substantial pain, and even lead to death. Schwannomas are difficult to treat because surgery involves cutting the affected nerves. There are currently no drug therapies, and due to the paucity of cell cultures and cell lines, there is a lack of molecular and cell biology data about schwannomas. The goal of this work was to better characterize a set of 20 tumors, to contribute information helpful in developing more targeted therapies. This involved loss of heterozygosity study using polymorphisms in or near the genes $N F 2, L Z T R 1$ and $S M A R C B 1$ to identify somatic deletions. DNA sequencing of $S M A R C B 1$ exons was also carried out to search for germline and somatic mutations. This work revealed several mutations that contribute new knowledge to the field.
\end{abstract}

\section{Introduction}

Schwannomas are benign Schwann cell tumors that can occur either sporadically or in the autosomal dominant conditions NF2 (neurofibromatosis type 2) or schwannomatosis (Ruggieri, Praticò, \& Evans, 2015; Kehrer-Sawatzki, Farschtschi, Mautner, \& Cooper, 2017). These can occur anywhere in the peripheral nervous system, including the spinal nerve roots, and the eighth cranial nerve in NF2 patients (vestibular schwannomas) that can cause deafness. NF2 is caused by germline mutations in the $N F 2$ gene, with tumors forming when the remaining normal gene copy is somatically mutated in a Schwann cell, consistent with the tumor suppressor two-hit phenomenon. In schwannomatosis, which is relatively newly described, two genes have been found to be involved so far having germline mutations: SMARCB1 and LZTR1, both near the 
NF2 gene on chromosome 22 (Huslebos et al., 2007; Piotrowski et al., 2014). Those tumors seem to be more complex, though, with early studies finding variable deletions of multiple genes including two or more of those genes (Hadfield et al., 2008). Schwannomas in schwannomatosis have a small risk of becoming malignant as well. Sporadic schwannomas are also common, and like in the inherited forms, can be difficult to treat because surgery (the only current therapy) involves cutting the nerves involved. Recent data suggest that mutations in NF2 and SMARCB1 genes contribute to some of these sporadic cases, but further data is needed to determine how many of these may be biologically similar to the inherited forms (Paganini et al., 2018). Schwannomas at nerve endings in the skin can be a cosmetic problem, but most occur on deeper nerves, causing functional problems that can even kill patients by impinging on vital structures. Thus, patients with NF2 and schwannomatosis often have a reduced lifespan (Kresak and Walsh, 2016). Schwannomas can also cause substantial pain (particularly in schwannomatosis), and this may relate to specific gene mutations (Jordan et al., 2018). There are no drug therapies to treat these tumors. Because there are very few tumor cell cultures or cell lines, there is a lack of molecular and cell biology data about schwannomas. Our lab has received a number of schwannoma tumor samples over the years and has been developing cell cultures and attempting to develop cell lines through immortalization of the early passages. This project's goal was to characterize these tumors and cells to better understand underlying genetic changes at the key genes, to provide information about the associated biochemical pathways. Such information will contribute to development of better targeted therapies for schwannomas.

Through an approved research protocol, our lab obtained a number of schwannomas and blood or buccal swabs from the same patients. Some subjects were known to have NF2 or schwannomatosis, and some were putatively sporadic cases. For one sporadic subject, a sample was available from each of two subsequent surgeries on the same tumor, and for another patient (with schwannomatosis) we also had more than one independent tumor.

It is known that these tumors, although benign, have a somatic mutation on the other gene copy for NF2, LZTR1, and/or SMARCB1 genes. Thus, with one gene copy mutated, as is the case when the mutation is inherited and constitutional, when the other gene copy mutated in a single Schwann cell, that cell initiates a tumor by the tumor suppressor two-hit mechanism (Knudson, 2001). Some of our cases had no family history, and it is thought that the tumor was sporadic, originating from one cell that by chance suffered mutations on both gene copies. 
One goal was to examine the NF2, LZTR1, and SMARCB1 genes to screen for somatic mutations, since identifying the precise mutations may shed light on biochemical mechanisms. A frequently-reported somatic mutation in Schwann cell tumors is a deletion of part or all of a gene; this can be detected by observing that the tumor DNA only has one allele present while the germline DNA has both copies at a DNA polymorphism in that gene (loss of heterozygosity analysis). Since it was possible that some of the "sporadic" cases were actually genetic in origin, with the most likely gene being $S M A R C B 1$, we also sequenced all of the $S M A R C B 1$ exons to discover any germline as well as subtle somatic mutations. For the NF2 and LZTR1 genes, loss of heterozygosity analysis was performed using polymorphisms in or near those genes as a rapid, effective screening tool to look for somatic deletions in the tumors, where subjects were heterozygous for one or more markers. Characterization of any germline or somatic mutations in schwannomas will add significantly to the field, contributing to discovery of phenotype-genotype correlations, and improved understanding of tumor biology.

\section{Methods}

\section{Samples}

DNA and RNA samples from blood, tumor and cell cultures had previoulsy been extracted in the lab from samples obtained under IRB approval. Standard methods had been used to extract nucleic acids (PureGene system for DNA (Qiagen); Trizol method for RNA (ThermoFisher)). The samples were coded to protect subject privacy. PCR dilutions of DNA samples were prepared (25-100 ng/uL) by dilution in sterile water. In a few cases, we only had access to germline material (usually from blood), not tumor, and in one case there was only tumor DNA available, not germline.

\section{Loss of Heterozygosity Analysis}

The first test conducted was a loss of heterozygosity (LOH) analysis in the tumor DNAs at the NF2 and LZTR1 genes to search for somatic deletions. Initially, the germline DNA was genotyped at known polymorphisms to find sites where the subject was heterozygous; at such sites the tumors were then genotyped to see if one allele was reduced or absent, indicating LOH. Native polyacrylamide gels of varying concentrations were used to separate alleles by electrophoresis to observe the genotypes after ethidium bromide staining to visualize the DNA fragments. 
PCR genotyping of NF2 IVS1TET (tetranucleotide repeat variant in NF2 intron 1, rs199935829) used PCR primers NF2IVS1TET-F: 5' CCTTATGCCATGTTCTTGTTGAAGG and NF2IVS1TET-R: 5' TGCAGCCTGGCAATAGAGCAAGA). PCR utilized standard conditions (HotStar, Qiagen) with $62^{\circ} \mathrm{C}$ annealing temperature to amplify a 220 base pair (bp) fragment spanning this region. PCR products were checked for specificity on a 1.1\% agarose gel. PCR products were then subjected to $12 \%$ polyacrylamide gel electrophoresis to resolve the fragments, with alleles differing by multiples of $4 \mathrm{bp}$.

PCR genotyping of NF2 rs3842713 employed primers NF2rs3842713-F 5' GCTGAGCATGAAGAACGGTTAG and NF2rs3842713-R: 5' AATGGGCACAACTCGGGTC, to genotype a 7 bp insertion/deletion polymorphism in intron 14 of the NF2 gene. Standard PCR conditions used a $60^{\circ} \mathrm{C}$ annealing temperature to produce the 158 bp fragment. PCR fragments were resolved on 12\% polacrylamide electrophoresis.

PCR genotyping of NF2 intron 13 single nucleotide polymorphism (SNP) rs731647 used forward primer NF2IVS13-F 5' GGCCTGCAACCTTCAGATAAAC, and reverse primer NF2IVS13-R 5' ATAAAACACCAGTGGGCCTTGT. Standard PCR conditions utilized a $58^{\circ} \mathrm{C}$ annealing temperature to amplify the 363 bp product. AccI (New England Biolabs) restriction digestion was used to specify alleles, as this enzyme cuts the A allele but not the T allele. Genotyping was done by separating digestion products by $8 \%$ polyacrylamide gel electrophoresis. Genotypes were called based on band patterns as follows: TT: 363 bp, AT: 363, 184, 179 bp, and AA: 184, 179 bp.

PCR genotyping of LZTR1 rs178280 (SNP in intron 1) utilized primers LZTR1rs178280-F 5' CTGAGGTGGGGTGAGGAGT and LZTR1rs178280-R 5' GGCATCTTTATAGGCCACCA. A $58^{\circ} \mathrm{C}$ annealing temperature was used in the PCR amplification of this 254 bp product. RsaI restriction digestion was used to distinguish the alleles, with the A allele being cut but not the $G$ allele. The fragments were resolved on $8 \%$ polyacrylamide gel electrophoresis, with genotypes called as follows: GG (uncut) $254 \mathrm{bp}$; AG (heterozygous) 254,158 and $87 \mathrm{bp}$; AA 158 and 87 bp. 


\section{Sequencing}

Sanger DNA sequencing of PCR-amplified SMARCB1 exons 1-9 was performed to screen for specific germline and/or somatic mutations in the coding region of this gene, as well as in immediate flanking intron sequences and some untranslated region sequence.

Primers (in parentheses) and conditions used for each exon:

- $\quad$ exon 1 (SMARCB1 ex.1-F: 5'-CCTCCTGATCCCTCGCAGC; SMARCB1 ex.1-R: 5'CGGGATACCTCGGAGCCGAT). Annealing temperature: $65^{\circ} \mathrm{C}$. PCR product: $140 \mathrm{bp}$. The forward strand was sequenced.

- $\quad$ exon 2 (SMARCB1 ex.2-F: 5'-CTGCGACCCTTATAATGAGC; SMARCB1ex.2-R: 5'GCGAGTGGTTTTGAAACAGG). Annealing temperature: $56^{\circ} \mathrm{C}$. PCR product: $213 \mathrm{bp}$. The reverse strand was sequenced.

- $\quad$ exon 3 (SMARCB1 ex.3-F: 5'-ACCAGCAGAGTGACCCAGTG; SMARCB1ex.3-R: 5'AGAGATGCCCTGGCCAGGAA). Annealing temperature: $65^{\circ} \mathrm{C}$. PCR product: $195 \mathrm{bp}$. Sequencing was done in both directions.

- $\quad$ exon 4 (SMARCB1 ex.4-F: 5'-GGATCAGGTCCTATACTGAC; SMARCB1 ex.4-R: 5'AACTAAGGCGGAATCAGCAC). Annealing temperature: $56^{\circ} \mathrm{C}$. PCR product: $225 \mathrm{bp}$. The reverse strand was sequenced.

- $\quad$ exon 5 (SMARCB1 ex.5-F: 5'-TTGCATACCTAGGGCTCCGG; SMARCB1 ex.5-R: 5'GCCCGACTGCCTTGTACCAT). Annealing temperature: $62^{\circ} \mathrm{C}$. PCR product: $201 \mathrm{bp}$. The forward strand was sequenced.

- $\quad$ exon 6 (SMARCB1 ex.6-F: 5'-TGGTGCAATCTCTTGGCATC; SMARCB1 ex.6-R: 5'TCAGTGCTCCATGATGACAC). Annealing temperature: $58^{\circ} \mathrm{C}$. PCR product: $277 \mathrm{bp}$. The reverse strand was sequenced.

- $\quad$ exon 7 (SMARCB1 ex.7-F: 5'-TGGGCTGCAAAAGCTCTAAC; SMARCB1ex.7-R: 5'CGCTCACACAGAGAAGTCTT). Annealing temperature: $56^{\circ} \mathrm{C}$. PCR product: $312 \mathrm{bp}$. The reverse strand was sequenced.

- $\quad$ exon 8 (SMARCB1ex.8-F: 5'-ATCCACTGGGTGCCAGCAGT; SMARCB1ex.8-R: 5'TCTGCCTGGAAAGCCAGGTG). Annealing temperature: $62^{\circ} \mathrm{C}$. PCR product: $313 \mathrm{bp}$. The reverse strand was sequenced. 
- exon 9 (SMARCB1ex.9-F: 5'-CCCTGTAGAGCCTTGGGAAG; SMARCB1ex.9-R: 5'GCCTCTGTCCTTGCCAGAAG). Annealing temperature: $60^{\circ} \mathrm{C}$. PCR product: $200 \mathrm{bp}$. The forward strand was sequenced.

For all DNA sequencing, standard PCR amplification was performed using the primers and annealing temperature specified above for each exon. The PCR products were purified (primers removed) using ExoSAPit treatment (ThermoFisher), and then quantified using Nanodrop spectrometry, to ensure a final concentration between $100-500 \mathrm{ng} / \mu \mathrm{L}$. The sequencing primer (one of the original PCR primers as specified above) was added to each PCR product in a SimpleSeq tube (Eurofins Genomics) and sent for fluorescence-based Sanger sequencing (Eurofins Genomics). Sequencing chromatogram data was returned and analyzed using Sequencher software (GeneCodes).

\section{Results}

For the LOH analysis, where the germline DNA was homozygous, the tumor was not genotyped since the situation was un-informative. Where we only had tumor DNA, if its genotype was homozygous with no sign of heterozygosity, it was also considered uninformative. Table 1 summarizes the LOH data where we obtained useful information about whether there may be a somatic deletion of that region in the tumor (N/A indicates homozygosity, un-informative). LOH screening at these polymorphisms in the NF2 and LZTR1 genes ( 3 and 1 SNP respectively) found NF2 somatic deletions in schwannomas $291 \mathrm{~T}$ and 1359T, consistent with those patients having an NF2 or schwannomatosis diagnosis (Figures 1 and 2). The deletion size is unclear but could extend well beyond the gene. Sample 373T was suspicious for $\mathrm{LOH}$ at $L Z T R 1$, but the intensities of the bands were not sufficiently different to be certain (thus, indicated as putative in Table 1). The rest of the samples, excluding 274 blood and tumor 285, showed heterozygous alleles without LOH or were completely un-informative, and thus were not included in Table 1.

Table 1: LOH screening at polymorphisms in NF2 and LZTR1

\begin{tabular}{|c|c|c|c|c|}
\hline Sample & NF2 IVS1 TET & $N F 2$ rs3842713 & NF2 rs731647 & LZTR1 rs 178280 \\
\hline $291 \mathrm{~T}$ & LOH & LOH & N/A & N/A \\
\hline $373 \mathrm{~T}$ & no $\mathrm{LOH}$ & N/A & N/A & no $\mathrm{LOH}$ \\
\hline $546 \mathrm{~T}$ & no $\mathrm{LOH}$ & N/A & N/A & N/A \\
\hline $1242 \mathrm{~T}$ & no LOH & N/A & N/A & N/A \\
\hline
\end{tabular}




\begin{tabular}{lcccc}
\hline $1359 \mathrm{~T}$ & N/A & N/A & LOH & LOH \\
ND7510S1T & N/A & N/A & no LOH & N/A \\
$2647 \mathrm{~T}$ & no LOH & N/A & N/A & no LOH \\
$2648 \mathrm{~T}$ & LOH & N/A & N/A & no LOH \\
\hline
\end{tabular}

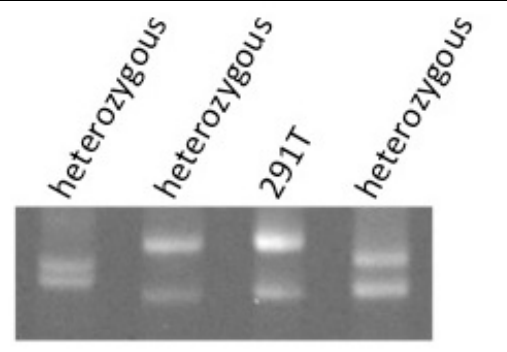

Figure 1. Photograph of $N F 2$ gene polymorphism IVS1 TET tetranucleotide repeat genotyping on polyacrylamide gel stained with ethidium bromide. Alleles are 4-, 8- or 12-bp different in size. There is evidence of $\mathrm{LOH}$ in sample 291T (third lane, alleles $12 \mathrm{bp}$ difference), with a substantially less-intense lower band relative to the upper band, and compared to the normal heterozygote in lane 2 with the same alleles.

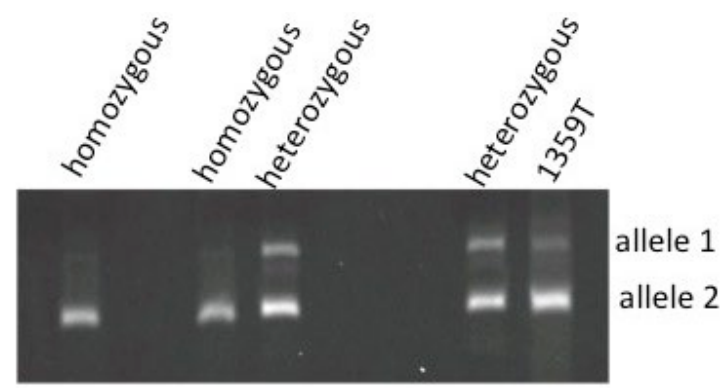

Figure 2. Photograph of ethidium-stained polacrylamide gel of LZTR1 rs178280 restriction digest showing LOH in tumor 1359T. The uncut (top) allele is substantially less-intense than the lower band in 1359T, relative to the other (normal) heterozygotes.

In exon DNA sequence analysis of the $S M A R C B 1$ gene, done for all tumors, (summarized in Table 2), sample 235T showed loss of heterozygosity for SMARCB1 exon 7 at polymorphism rs5844569, with one allele's peaks substantially lower than the other (Figure 3). Several other samples were heterozygous for that polymorphism with no sign of LOH ("no LOH" in Table 2). Sample 2648T also showed reduction of one allele in exon 7, at another SNP in that exon (rs2229354). Samples for all other exons showed normal sequences (N).

Table 2. Sequence data for $S M A R C B 1$ exons 1-9

\begin{tabular}{lccccccccc}
\hline Sample & ex. 1 & ex. 2 & ex. 3 & ex. 4 & ex. 5 & ex. 6 & ex. 7 & ex. 8 & ex. 9 \\
\hline 93 & $\mathrm{~N}$ & $\mathrm{~N}$ & $\mathrm{~N}$ & $\mathrm{~N}$ & $\mathrm{~N}$ & $\mathrm{~N}$ & no LOH & $\mathrm{N}$ & $\mathrm{N}$ \\
$235 \mathrm{~T}$ & $\mathrm{~N}$ & $\mathrm{~N}$ & $\mathrm{~N}$ & $\mathrm{~N}$ & $\mathrm{~N}$ & $\mathrm{~N}$ & LOH $^{\mathbf{1}}$ & $\mathrm{N}$ & $\mathrm{N}$ \\
274 & $\mathrm{~N}$ & $\mathrm{~N}$ & $\mathrm{~N}$ & $\mathrm{~N}$ & $\mathrm{~N}$ & $\mathrm{~N}$ & $\mathrm{~N}^{2}$ & $\mathrm{~N}$ & $\mathrm{~N}$ \\
\hline
\end{tabular}




\begin{tabular}{|c|c|c|c|c|c|c|c|c|c|}
\hline $285 \mathrm{~T}$ & $\mathrm{~N}$ & $\mathrm{~N}$ & $\mathrm{~N}$ & $\mathrm{~N}$ & $\mathrm{~N}$ & $\mathrm{~N}$ & $\mathrm{~N}^{2}$ & $\mathrm{~N}$ & $\mathrm{~N}$ \\
\hline $373 \mathrm{~T}$ & $\mathrm{~N}$ & $\mathrm{~N}$ & $\mathrm{~N}$ & $\mathrm{~N}$ & $\mathrm{~N}$ & $\mathrm{~N}$ & no $\mathrm{LOH}^{1}$ & $\mathrm{~N}$ & $\mathrm{~N}$ \\
\hline $469 \mathrm{~T}$ & $\mathrm{~N}$ & $\mathrm{~N}$ & $\mathrm{~N}$ & $\mathrm{~N}$ & $\mathrm{~N}$ & $\mathrm{~N}$ & no LOH & $\mathrm{N}$ & $\mathrm{N}$ \\
\hline $541 \mathrm{~T}$ & $\mathrm{~N}$ & $\mathrm{~N}$ & $\mathrm{~N}$ & $\mathrm{~N}$ & $\mathrm{~N}$ & $\mathrm{~N}$ & $\mathrm{~N}^{2}$ & $\mathrm{~N}$ & $\mathrm{~N}$ \\
\hline $546 \mathrm{~T}$ & $\mathrm{~N}$ & $\mathrm{~N}$ & $\mathrm{~N}$ & $\mathrm{~N}$ & $\mathrm{~N}$ & $\mathrm{~N}$ & no $\mathrm{LOH}$ & $\mathrm{N}$ & $\mathrm{N}$ \\
\hline $1242 \mathrm{~T}$ & $\mathrm{~N}$ & $\mathrm{~N}$ & $\mathrm{~N}$ & $\mathrm{~N}$ & $\mathrm{~N}$ & $\mathrm{~N}$ & $\mathrm{~N}^{2}$ & $\mathrm{~N}$ & $\mathrm{~N}$ \\
\hline $1359 \mathrm{~T}$ & $\mathrm{~N}$ & $\mathrm{~N}$ & $\mathrm{~N}$ & $\mathrm{~N}$ & $\mathrm{~N}$ & $\mathrm{~N}$ & no $\mathrm{LOH}$ & $\mathrm{N}$ & $\mathrm{N}$ \\
\hline $1778 \mathrm{~T}$ & $\mathrm{~N}$ & $\mathrm{~N}$ & $\mathrm{~N}$ & $\mathrm{~N}$ & $\mathrm{~N}$ & $\mathrm{~N}$ & no $\mathrm{LOH}$ & $\mathrm{N}$ & $\mathrm{N}$ \\
\hline $1935 \mathrm{~T}$ & $\mathrm{~N}$ & $\mathrm{~N}$ & $\mathrm{~N}$ & $\mathrm{~N}$ & $\mathrm{~N}$ & $\mathrm{~N}$ & no $\mathrm{LOH}$ & $\mathrm{N}$ & $\mathrm{N}$ \\
\hline $2127 \mathrm{~T}$ & $\mathrm{~N}$ & $\mathrm{~N}$ & $\mathrm{~N}$ & $\mathrm{~N}$ & $\mathrm{~N}$ & $\mathrm{~N}$ & no $\mathrm{LOH}$ & $\mathrm{N}$ & $\mathrm{N}$ \\
\hline $2647 \mathrm{~T}$ & $\mathrm{~N}$ & $\mathrm{~N}$ & $\mathrm{~N}$ & $\mathrm{~N}$ & $\mathrm{~N}$ & $\mathrm{~N}$ & no $\mathrm{LOH}$ & $\mathrm{N}$ & no $\mathrm{LOH}$ \\
\hline $2648 \mathrm{~T}$ & $\mathrm{~N}$ & $\mathrm{~N}$ & $\mathrm{~N}$ & $\mathrm{~N}$ & $\mathrm{~N}$ & $\mathrm{~N}$ & $\mathbf{L O H}^{1}$ & $\mathrm{~N}$ & $\mathrm{~N}$ \\
\hline
\end{tabular}

Note: N: normal sequence; LOH indicates loss of heterozygosity; "no LOH" indicates germline heterozygosity at a polymorphism with no evidence of $\mathrm{LOH}$ in the tumor $1=$ informative at rs5844569. 2 = informative at rs2229354. Exon 9 SNP detected was rs 144863210.

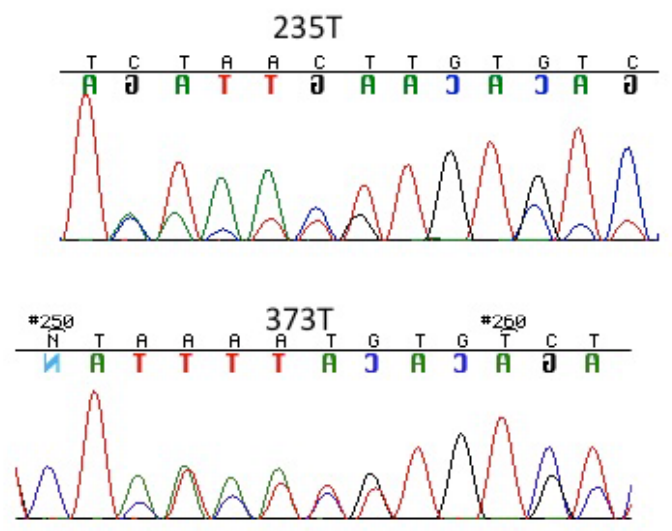

Figure 3. Sequencing chromatograms from SMARCB1 ex. 7 sequencing of tumors $235 \mathrm{~T}$ (top) and 373T (bottom). Both "doublet" patterns indicate that the patients are germline heterozygous for a 2-bp insertion/deletion polymorphism in intron 7 (rs5844569), with tumor 235T showing LOH (one allele's peaks are lower than the other allele) compared to 373 T's peaks, which are much closer in height (no LOH).

\section{Discussion}

Schwannomas are benign Schwann cell tumors that can appear either sporadically or as a result of dominant genetic tumor syndromes NF2 or schwannomatosis. In the past, there was also confusion in pathological diagnosis based on histology, with some neurofibromas (benign Schwann cell tumors seen in neurofibromatosis type 1) being called "schwannomas." In addition, sometimes a patient is not recognized as having NF2 or schwannomatosis constitutionally, with 
healthcare providers believing a schwannoma is sporadic with no genetic predisposition. Because of these reasons, we chose to investigate the three most implicated genes on chromsome 22 in a set of schwannomas: NF2, SMARCB1, and LZTR1. In the inherited situations, there is a germline mutation in one gene, with somatic mutation inactivating the other allele of that gene (plus at least one other gene for schwannomatosis). Examination of these genes was done with the goal of identifying whether any "sporadic" tumors might be associated with germline mutations, and to determine which genes showed evidence of somatic mutation as well. Knowledge of specific mutations is useful for the field in understanding diagnostics, pathogenesis and mutation effect, which directly informs development of targeted therapies for abnormalities in those pathways (e.g. Fuse et al., 2017).

Among the DNA samples examined from 17 patients, LOH (somatic deletion) at one or more genes was identified in 4 tumors $(235 \mathrm{~T}, 291 \mathrm{~T}, 1359 \mathrm{~T}$, and 2648T). The most useful marker was NF2 IVS1 TET, a repeat polymorphism with 4 known alleles (normal, +4 (one TTTG repeat), +8 (2 TTTG repeats), and +12 (3 TTTG repeats)). Since this polymorphism, with more than 2 alleles, has greater heterozygosity than simple two-allele single nucleotide substitutions, most subjects were informative for the $\mathrm{LOH}$ analysis. $S M A R C B 1$ sequencing did not reveal any specific mutations, but did uncover LOH in two samples. Subject 291 has schwannomatosis and the tumor was found to have $\mathrm{LOH}$ in the $N F 2$ gene (uninformative elsewhere). This subject was known to have a SMARCB1 germline mutation in exon 9, so we have identified the tumor's somatic mutation as being a large deletion likely spanning both $N F 2$ and SMARCB1. By the restriction digest assay, tumor 373 T was questionable for a LZTR1 somatic deletion, but sequencing of that PCR product showed that both alleles were present in approximately equal quantities, ruling out somatic deletion. Subject 1359, who does not have a specific diagnosis, had a tumor that showed LOH at NF2 and LZTR1. So future analyses will screen for second mutations in those genes to see if the two-hit mechanism is fulfilled and whether the patient has a constitutional mutation. Patient 235 has NF2, and the tumor showed LOH in the SMARCB1 gene; since previous data had found $N F 2 \mathrm{LOH}$, this indicates there is a large somatic chromosomal deletion spanning both genes in that tumor. The 2648 schwannoma was found to have LOH in SMARCB1. Neither the primary tumor nor tumor Schwann cell culture from schwannoma 2647 showed LOH or a SMARCB1 mutation. Thus, we have found different, independent mutations in these patients and tumors. 
One limitation is that $\mathrm{LOH}$ analysis can only detect deletions, and relies on patients being heterozygous, so it does not rule out mutations in other parts of those genes, or in patients whose germline genotypes were homozygous. Also, some samples (since most DNA samples were derived from primary tissue) may have variable admixture from non-tumor tissues (blood vessels, connective tissue) that would increase background sufficiently to make mutation detection very difficult, such as masking $\mathrm{LOH}$. In the future, in addition to the follow-up experiments mentioned above, we will continue to search for more changes in these genes in the tumors. DNA sequencing of NF2 and LZTR1 will screen for the small mutations. Also, sequencing of cDNA is one approach to finding mutations that may not be detectable at the DNA level, such as splicing errors (for all three genes). We could also examine DNA methylation as an alternative, "epi-mutation" silencing these genes. This latter phenomenon would be another way to inactivate the gene, without an actual DNA change. If the opportunity arises, we would also like to obtain exome sequence data from the tumors, to identify what, if any, other genes are altered and may be contributing to the tumor phenotype. The data produced here suggest that $N F 2, L Z T R 1$, and SMARCB1 are all excellent candidate genes to examine for somatic (and possibly germline) mutations in Schwann cell tumors, regardless of whether the subject is known to have NF2 or schwannomatosis. By understanding exactly how these genes are mutated in these tumors, we can predict functional effects on their proteins (from amino acid substitution to deletion), which is important in understanding the tumor mechanisms so that specific therapies can be designed or chosen.

\section{Acknowledgements}

The authors thank the University of Florida's Department of Molecular Genetics and Microbiology, the College of Medicine, and the Center for Undergraduate Research for A.M's opportunity to participate in this research through the University Scholars Program. We also thank the subjects and their physicians for providing research samples. The supplies for this work were funded by a Children's Tumor Foundation contract to M.R.W.

\section{References}

Fuse MA, Plati SK, Burns SS, Dinh CT, Bracho O, Yan D, Mittal R, Shen R, Soulakova JN, Copik AJ, Liu XZ, Telischi FF, Chang LS, Franco MC, Fernandez-Valle C. Combination therapy with cmet and src inhibitors induces caspase-dependent apoptosis of merlin-deficient schwann cells and suppresses growth of schwannoma cells. Mol Cancer Ther. 2017 Nov;16(11):2387-239.

Hadfield KD, Newman WG, Bowers NL, Wallace A, Bolger C, Colley A, McCann E, Trump D, Prescott T, Evans DG. Molecular characterization of SMARCB1 and NF2 in familial and sporadic schwannomatosis. J Med Genet. 2008 Jun;45(6):332-339. 
Hulsebos TJM, Plomp AS, Wolterman RA, Robanus-Manndag EC, Baas F, Wesseling P. Germline mutation of INI1/SMARCB1 in familial schwannomatosis. Am J Hum Genet. 2007

April;80:805-810.

Jordan JT, Smith MJ, Walker JA, Erdin S, Talkowski ME, Merker VL, Ramesh V, Cai W, Harris GJ, Bredella MA, Seljo M, Suuberg A, Gusella JF, Plotkin SR. Pain correlated with germline mutation in schwannomatosis. Medicine. 2018 97:5(e9717).

Kehrer-Sawatzki H, Farschtschi S, Mautner VF, Cooper DN. The molecular pathogenesis of schwannomatosis, a paradigm for the co-involvement of multiple tumour suppressor genes in tumorigenesis. Hum Genet. 2017 Feb;136(2):129-148.

Knudson, AG. Two Genetic Hits (More or Less) To Cancer. Nature Reviews. 2001 Nov;1(1):157-158.

Kresak JL, Walsh M. Neurofibromatosis: a review of NF1, NF2, and schwannomatosis. J Pediatr Genet. 2016 Jun;5(2):98-104.

Paganini I, Capone GL, Vitte J, Sestini R, Putignano AL, Giovannini M, Papi L. Double somatic SMARCB1 and NF2 mutations in sporadic spinal schwannomas. J Neurooncol. 2018 Mar;137(1):33-38.

Piotrowski A, Xie J, Liu YF, Poplawski AB, Gomes AR, Madanecki P, Fu C, Crowley MR, Crossman DK, Armstrong L, Babovic-Vuksanovic D, Bergner A, Blakely JO, Blumenthal AI, Daniels MS, Feit H, Gardner K, Hurst S, Kobelka C, Lee C, Nagy R, Rauen KA, Slopis JM, Suwannarat P, Westman JA, Zanko A, Korf BR, Messiaen LM. Germline loss-of-function mutations in LZTR1 predispose to an inherited disorder of multiple schwannomas Nat Genet. 2014 Feb;46(2):182-187.

Ruggieri M, Praticò AD, Evans DG. Diagnosis, Management, and Therapeutic Options in Childhood NF2 and Related Forms. Semin Pediatr Neurol. 2015 Dec;22(4):240-58. 